\title{
Influencia de las condiciones de preparación en la luminiscencia de recubrimientos de sílice pura
}

\author{
M.A. GARCÍA ${ }^{1}$, S.E. PAJE ${ }^{1}$, J. LLOPIS ${ }^{1}$ Y M.A. VILLEGAS ${ }^{2}$ \\ ${ }^{1}$ Departamento de Física de Materiales. Fac. de CC. Físicas. \\ UCM. Av. Complutense s/n. 28040 Madrid. Fax: 91394547 / e-mail: jllopis@eucmax.sim.ucm.es \\ ${ }^{2}$ Departamento de Vidrios. Instituto de Cerámica y Vidrio, CSIC. \\ Antigua Ctra. Valencia Km. 24,300. 28500 Arganda del Rey. Madrid.
}

\begin{abstract}
Los recubrimientos de $\mathrm{SiO}_{2}$ preparados mediante la técnica sol-gel, presentan una luminiscencia en la zona UV-Visible del espectro con un máximo centrado en torno a 3,4 eV $(365 \mathrm{~nm})$. El espectro de emisión puede descomponerse en dos bandas de perfil gausiano, centradas en 3,35 eV $(370 \mathrm{~nm})$ y 3,0 eV $(415 \mathrm{~nm})$, respectivamente. El espectro de excitación correspondiente a esta emisión presenta una banda centrada en 3,85 eV (320 nm). Con objeto de clarificar el origen de esta luminiscencia se ha realizado el estudio según los precursores utilizados en la preparación de los recubrimientos y el efecto que los tratamientos térmicos de densificación tienen sobre la luminiscencia. Asimismo, se ha comprobado que la presencia de materia orgánica en los recubrimientos antes de ser densificados modifica las propiedades luminiscentes de los mismos. Por tanto, ciertas variaciones en el espectro de la luminiscencia podrían interpretarse como indicativas de un cierto cambio en el contenido de restos orgánicos en el recubrimiento, así como de posibles variaciones del contenido en exceso de oxígeno.
\end{abstract}

Palabras clave: Recubrimientos, luminiscencia, silice.

Influence of preparating conditions on the luminescence of pure silica coatings

$\mathrm{SiO}_{2}$ coatings prepared by sol-gel technique show an UV-Visible luminescence with a maximum centered at about $3.4 \mathrm{eV}$ (365 $\mathrm{nm})$. The emission spectrum can be decomposed into two Gaussian shape bands, centered at $3.35 \mathrm{eV}(370 \mathrm{~nm}) \mathrm{and} 3.0 \mathrm{eV}$ $(415 \mathrm{~nm})$, respectively. The corresponding excitation spectrum shows a band centered at $3.85 \mathrm{eV}(320 \mathrm{~nm})$. In order to clarify the origin of that luminescence, a study as a function of precursors used for coatings preparation was done, as well as a function of thermal treatments carried out during densification. Moreover, the presence of organic matter in the coatings prior to densification, modifies their luminescent properties. So that, some variations of the luminescence spectrum could be understood as the result of both changes in the residual organics of coatings and variations in the surplus oxygen content.

Keywords: Coatings, luminescence, silica.

\section{INTRODUCCION}

La sílice es una de las sustancias más abundantes de la corteza terrestre cuyas propiedades fisico-químicas la han convertido desde tiempos remotos en un material de gran importancia tecnológica. Sin embargo, para la mayoría de las aplicaciones actuales no se puede utilizar tal y como se encuentra en la naturaleza, ya que es necesario cierto grado de pureza y, aunque existen algunas técnicas de refino, han sido los procesos de síntesis desarrollados a partir de precursores puros los que han marcado el camino para obtener la sílice en sus mayores grados pureza. Entre estos métodos de síntesis, los denominados vía sol-gel presentan ventajas en cuanto a la facilidad de controlar sus composiciones, homogeneidad del producto y baja temperatura de procesado; es decir economía, lo que los hacen muy atractivos desde el punto de vista industrial [1]. Asimismo, el método permite obtener recubrimientos sin agrietamiento con espesores comprendidos entre 100 y 1000 $\mathrm{nm}$ aproximadamente. Es un hecho bien conocido que gran parte de los defectos que se generan en la sílice dependen de los precursores utilizados y del método de preparación, siendo muchos de estos defectos centros ópticamente activos [2-4]. Por ello, es fundamental llevar a cabo una buena caracteriza- ción óptica de los recubrimientos de sílice pura, tanto para analizar sus posibles efectos sobre las propiedades ópticas finales del material compuesto, como para estudiar la relación de la microestructura con dichas propiedades ópticas. Puesto que es posible obtener un recubrimiento de sílice vía sol-gel a través de distintos procedimientos, la determinación de cómo puedan éstos influir en propiedades concretas como la luminiscencia es de gran interés práctico. En este sentido cabe destacar la utilización de matrices de sílice pura para hospedar iones metálicos y moléculas orgánicas con propiedades luminiscentes. En el primer caso, como fase previa a la precipitación en forma de nanopartículas que, como bien se sabe, confieren al recubrimiento nuevas propiedades mecánicas, ópticas, magnéticas y especialmente de una respuesta no lineal a los campos electromagnéticos, de gran interés aplicado [5,6]. Y en segundo lugar, en aplicaciones propiamente luminescentes [7-9], láseres [10] y sensores ópticos [11,12], entre otras.

Por tanto, el principal objetivo de este trabajo, es el estudio de la luminiscencia de recubrimientos de sílice preparados vía sol-gel y del efecto que las distintas condiciones de preparación puedan tener en la misma. 


\section{EXPERIMENTAL}

\subsection{Preparación de los recubrimientos}

Los recubrimientos de sílice pura se obtuvieron a partir de soles elaborados con distintos precursores:

1.- $\mathrm{Si}(\mathrm{OCH} 3)_{4}$ (TMOS),

2.- $\mathrm{Si}\left(\mathrm{OCH}_{2} \mathrm{CH}_{3}\right)_{4}$ (TEOS),

3.- $\mathrm{TMOS}+\mathrm{SiCH}_{3}\left(\mathrm{OCH}_{2} \mathrm{CH}_{3}\right)_{3}$ (MTES) $(50+50 \%$ molar $)$

4.- TEOS+MTES (50+50\% molar).

La relación molar de alcóxido(s): $\mathrm{H}_{2} \mathrm{O}$ fue de 1:3, incorporandose ésta como una disolución de $\mathrm{HCl} \mathrm{0,1} \mathrm{N}$, a fin de catalizar la hidrólisis de los alcóxidos. Se utilizó metanol en una proporción de 7 moles por mol de alcóxido(s), lo que da lugar a concentraciones finales de los soles de 123, 107, 126 y $114 \mathrm{~g}$ de sílice por litro de sol, respectivamente para cada uno de los posibles precursores mencionados. El $\mathrm{pH}$ final de los soles fue aproximadamente de 3 .

Los recubrimientos se depositaron a temperatura ambiente por la técnica de inmersión-extracción utilizando como sustratos portas de microscopio de vidrio sódico cálcico. Las velocidades de extracción se variaron entre 5 y $30 \mathrm{~cm} \mathrm{~min}^{-1}$. Algunas muestras se prepararon en una cámara de atmósfera controlada de nitrógeno a $25^{\circ} \mathrm{C}$ con contenido de humedad relativa por debajo del $6 \%$.

Las muestras se secaron a temperatura ambiente algunos minutos y después un conjunto de ellas se calentó a $60^{\circ} \mathrm{C}$ en aire durante $24 \mathrm{~h}$, otro conjunto lo hizo a $250^{\circ} \mathrm{C}$ durante $5 \mathrm{~h}$ y finalmente un tercer conjunto se calentó $500^{\circ} \mathrm{C}$ durante $1 \mathrm{~h}$. Por otro lado, se llevaron a cabo también tratamientos en atmósfera reductora de $\mathrm{N}_{2}-\mathrm{H}_{2}$ (90-10\% en volumen), con el fin de evaluar la posible influencia que la ausencia de oxígeno pudiera tener sobre la luminscencia de los recubrimientos.

Todas las muestras preparadas presentan un aspecto transparente e incoloro antes y después de los tratamientos térmicos.

\subsection{Equipo experimental}

Los espectros de absorción se registraron en el intervalo de 200 a $800 \mathrm{~nm}$ con un espectrofotómetro Shimazdu 3100 al que se le acopló una esfera integradora. Sin embargo, y debido a que el sustrato de vidrio sódico cálcico sobre el que se depositaron los recubrimientos tiene el borde de absorción óptica en $4 \mathrm{eV}$, el espectro de absorbancia del recubrimiento sólo se pudo medir para energías inferiores a dicho borde de absorción. En este intervalo, no se observó ninguna banda de absorción. Las medidas de fotoluminiscencia se llevaron a cabo con un espectrofluorímetro Perkin-Elmer LS-5. Algunas medidas de caída temporal de la luminiscencia se realizaron en el Departamento de Química Física de la Universidad de Venecia, utilizando como fuente de excitación un láser de Nd:YAG, cuadruplicando la frecuencia a fin de obtener luz de $266 \mathrm{~nm}$ de longitud de onda. Más detalles sobre el equipo experimental se han recogido en [13].

\subsection{Medida del espesor de los recubrimientos}

El espesor de los recubrimientos se midió a través del espectro de reflectancia obtenido con el espectrofotómetro de doble haz Shimadzu 3100. El método se basa en que, por una parte los recubrimientos son transparentes en la zona UV próximo-


Figura 1. (a) Al incidir luz sobre el recubrimiento, una parte se refleja en la superficie del mismo, y otra es refractada y posteriormente reflejada en la intercara recubrimiento-sustrato. La interferencia de ambas reflexiones, produce un espectro de reflectancia modulado como se muestra en (b).

visible del espectro y, por otra, en que tienen un espesor del mismo orden de magnitud que la longitud de onda con que se ilumina. Esto da lugar a que se produzcan franjas de interferencia entre la luz reflejada por la superficie plana del recubrimiento y la reflejada en la intercara recubrimiento-sustrato, tal como se muestra en la figura 1a. En este caso el espectro de reflectancia aparece modulado con un patrón de máximos y mínimos. La figura $1 \mathrm{~b}$ muestra un espectro de reflectancia típico de uno de los recubrimientos estudiados. A partir del espectro de reflectancia y teniendo en cuenta las posiciones de los máximos y mínimos, se puede determinar el espesor de los recubrimientos. Para ello se numeran los máximos con números enteros y los mínimos con semienteros como muestra la figura $1 \mathrm{~b}$. El espesor del recubrimiento puede evaluarse considerando dos o más máximos (adyacentes o no), dos o más mínimos o un máximo y un mínimo. Entonces la ecuación que da el espesor es: 


$$
\mathrm{d}=\frac{\Delta \mathrm{m}}{2 \sqrt{\mathrm{n}^{2^{2}}-\operatorname{sen}^{2} \theta}} \frac{\lambda_{1} \times \lambda_{2}}{\lambda_{2}-\lambda_{1}}
$$

donde $\Delta m=m_{1}-\mathrm{m}_{2}$, siendo $m_{1}$ y $m_{2}$ los números de orden correspondientes a los máximos o mínimos considerados, $\lambda_{1}$ y $\lambda_{2}$, las longitudes de onda de los mismos, $n$ el índice de refracción del recubrimiento y $\theta$ el ángulo de incidencia del haz sobre el recubrimiento (usualmente fijo para cada espectrofotómetro).

En el espectro de reflectancia que se muestra en la figura $1 \mathrm{~b}$, los máximos se encuentran en $503 \mathrm{~nm}$ (1) y $342 \mathrm{~nm}(2)$, y los mínimos en 668 nm (1/2) y 407 nm (3/2). Para el espectrofotómetro utilizado, el ángulo de incidencia es de $8^{0} \mathrm{y}$ asumiendo un índice de refracción para la sílice de $n=1,47$, el espesor del recubrimiento resulta ser de $360 \pm 15 \mathrm{~nm}$.

A efectos comparativos, se midió también el espesor de algunos recubrimientos utilizando un perfilómetro Taylor-Hobson. En todos los casos las diferencias relativas entre espesores medios medidos ópticamente y mecánicamente fue inferior al $10 \%$. Las diferencias aparecen debido a que el perfilómetro da un valor del espesor local del recubrimiento, mientras que la medida óptica da un espesor promedio de toda la zona iluminada. Una ventaja añadida del método óptico es que da información cualitativa sobre la homogeneidad del espesor del recubrimiento. Así, para un recubrimiento con espesor homogéneo, los máximos y mínimos en el espectro de reflectancia se definirán claramente, mientras para un recubrimiento con espesor irregular el patrón de interferencia quedará menos marcado. Una posible limitación del método óptico surge cuando existen bandas de absorción o/y de luminiscencia debidas al material que distorsionan fuertemente el espectro de reflectancia. En estos casos la medida del espesor se intenta realizar escogiendo una zona del espectro de reflectancia libre de estos efectos.

\section{RESULTADOS}

Todos los recubrimientos estudiados presentan una emisión característica de fotoluminiscencia en la zona UV-VIS del espectro cuando son excitados con luz ultravioleta. La figura 2a muestra un espectro de emisión típico, formado por una banda ancha con el máximo en 3,35 eV (370 nm). Esta banda puede descomponerse en suma de dos componentes de perfil gausiano, una más intensa, con el máximo en 3,40 eV (365 nm) y una anchura a la mitad del máximo (semianchura) de $0,4 \mathrm{eV}$; y otra, de menor intensidad, con el máximo en $3,0 \mathrm{eV}(415 \mathrm{~nm})$ y con una semianchura de 0,5-0,6 eV, como se muestra en la figura 2a. El espectro de excitación correspondiente a la emisión en 3,35 $\mathrm{eV}(370 \mathrm{~nm})$ se presenta en la figura $2 \mathrm{~b}$ y está formado por una banda ancha con el máximo en 3,85 eV (320 nm) y una semianchura de $0,4 \mathrm{eV}$. La naturaleza compuesta de este espectro no pudo manifestarse por deconvolución tan claramente como en el caso del espectro de emisión. No obstante se observan variaciones, tales como la correspondiente a la posición del máximo con la energía de la emisión del orden de $70 \mathrm{meV}$. Así,
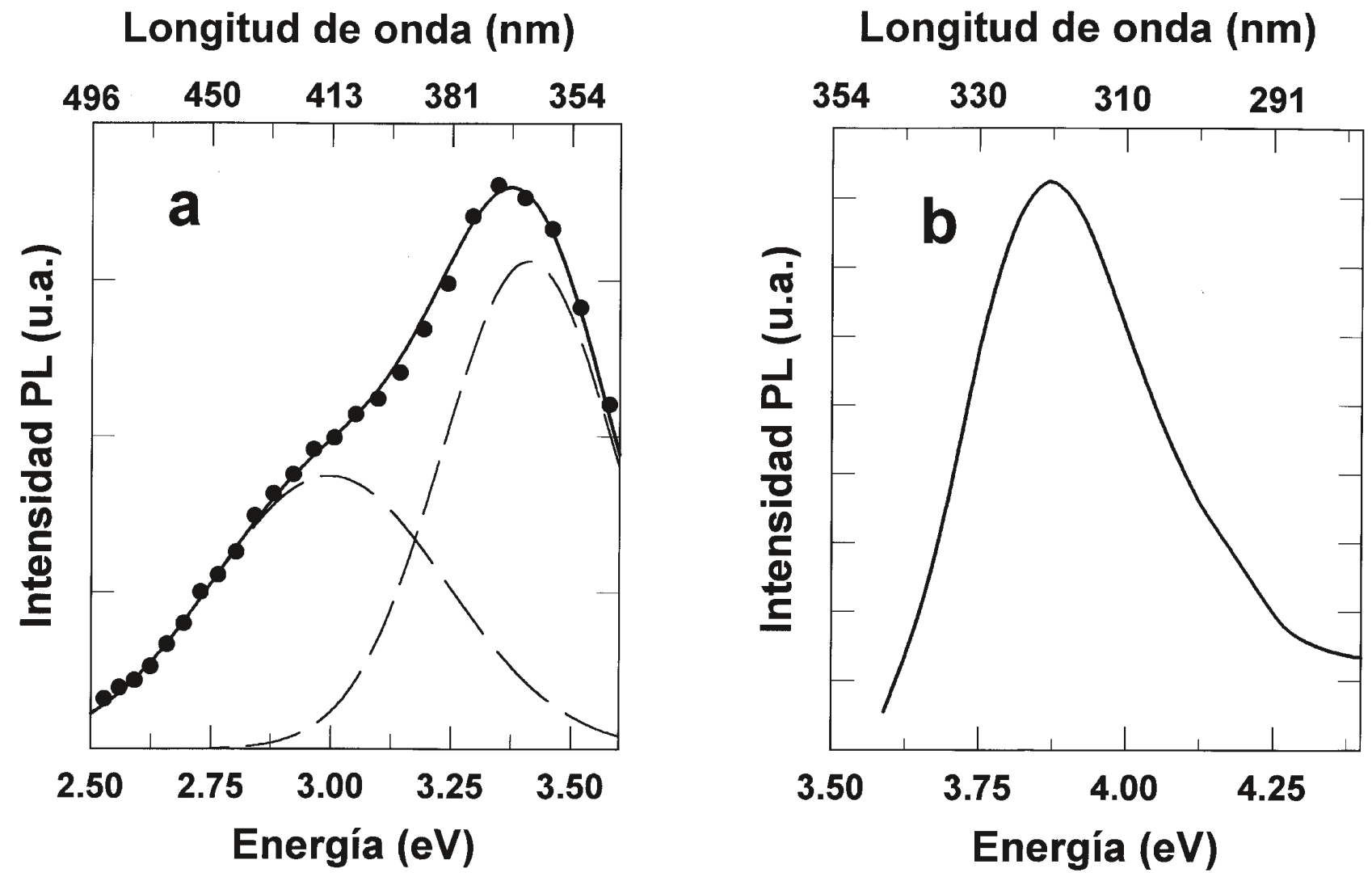

Figura 2. (a) Espectro de emisión típico de un recubrimiento de $\mathrm{SiO}_{2}$ al ser excitado con luz de 3,85 eV (320 nm). El resultado experimental (•) puede ser ajustado (línea continua) a la suma de dos bandas de perfil gausiano (línea discontinua); (b) Espectro de excitación del mismo recubrimiento correspondiente a la emisión de 3,35 eV (370 nm). 
tal como se muestra en la figura 3, a medida que se incrementa la energía de la emisión (disminución de la longitud de onda), se aprecia que el máximo de la banda de excitación se desplaza también hacia mayores energías (menores longitudes de onda). Análogamente, al aumentar la energía de excitación se produce un desplazamiento de la banda de emisión hacia mayores energías.

Un hecho importante que hemos observado sobre la intensidad de la emisión de 3,4 eV (365 nm) es que no depende del espesor del recubrimiento. Este comportamiento difiere claramente del observado en otras emisiones activadas por iones metálicos para este mismo tipo de recubrimientos. Así, para las emisiones de los recubrimientos dopados con pequeñas concentraciones de plata y cobre en forma de iones $\mathrm{Ag}^{+} \mathrm{y} \mathrm{Cu}^{+}$, respectivamente, se observa un aumento lineal de la intensidad de la emisión con el espesor del recubrimiento [9]. Por el contrario y como se ha mencionado anteriormente, para recubrimientos de sílice pura con espesores variables entre 150 y $500 \mathrm{~nm}$, la intensidad de la emisión de 3,4 eV (365 nm) es prácticamente la misma.

Los espectros de luminiscencia para recubrimientos preparados a partir de distintos precursores de sílice y excitados bajo las mismas condiciones no presentan variaciones apreciables. En la figura 4 se puede visualizar cómo, en efecto, la forma del espectro de emisión no cambia cuando se comparan entre sí los espectros procedentes de recubrimientos obtenidos con los diferentes precursores y sometidos a un mismo tratamiento térmico. Además, si se comparan los espectros de los recubrimientos secados a temperatura ambiente con los de los recubrimientos calentados posteriormente a $60^{\circ} \mathrm{C}$ durante $24 \mathrm{~h}$ y con los sometidos a $250^{\circ} \mathrm{C}$ durante $5 \mathrm{~h}$, no se observan variaciones apreciables. Sin embargo, ésto no ocurre con los espectros de los recubrimientos densificados a $500^{\circ} \mathrm{C}$ en aire. En éstos el cociente entre la intensidad de la componente de $3,0 \mathrm{eV}(415 \mathrm{~nm})$ y la correspondiente a la componente de $3,4 \mathrm{eV}(365 \mathrm{~nm})$ es 0,6 ; mientras que para el resto de recubrimientos la razón es de 0,7. En la figura 3 c se puede observar la variación relativa de intensidades de las dos componentes de la banda de emisión en función de la longitud de onda de excitación, para las muestras densificadas a $500^{\circ} \mathrm{C}$. La relación presenta el mínimo al excitar con 3,85 $\mathrm{eV}(320 \mathrm{~nm})$

Por otro lado, se observa que en general los recubrimientos preparados en la cámara de atmósfera de nitrógeno tienen la componente principal de 3,4 eV (365 nm) menos intensa que aquéllos preparados en aire. Análogamente, para los recubrimientos densificados en atmósfera reductora $\left(10 \% \mathrm{H}_{2}-90 \% \mathrm{~N}_{2}\right)$, la intensidad del espectro de emisión también es menor que la de aquellos densificados en aire.

Los análisis de la fotoluminiscencia en recubrimientos de sílice que contienen pequeñas cantidades de metales nobles $(\mathrm{Ag}, \mathrm{Au} \mathrm{y} \mathrm{Cu})$ muestran esencialmente esta misma emisión luminiscente a $3,4 \mathrm{eV}$. Únicamente en el caso de los recubrimientos con plata en los que ésta se encuentra en forma $\mathrm{de}{ }^{+}$, el espectro es diferente. La variación en este caso se debe a un solapamiento de la banda de emisión de la matriz de sílice con la emisión debida a los iones $\mathrm{Ag}^{+}$. En particular se observa que al ser excitados con luz de 5,43 eV (228 nm), los iones $\mathrm{Ag}^{+}$en la sílice emiten luz de 3,85 eV (320 nm), que corresponde a la energía de excitación de la luminiscencia a 3,4 eV (365 nm) [9]. La presencia de nanopartículas metálicas tampoco parece afectar a la emisión característica de 3,4 eV (365 nm) del recubrimiento.

La vida media de los procesos luminiscentes observados resultó estar por debajo del límite de detección del equipo experimental utilizado $(10 \mu \mathrm{s})$. No obstante, se pudo medir la caída temporal de la emisión de $3 \mathrm{eV}(410 \mathrm{~nm})$ excitando con luz de


Figura 3. (a) Evolución del máximo de emisión en función de la longitud de onda de excitación para los recubrimientos de sílice. (b) Evolución del máximo de excitación en función de la longitud de onda de emisión para los recubrimientos de sílice. (c) Variación relativa de intensidades de las dos componentes de la banda de emisión en función de la longitud de onda de excitación, para los recubrimientos tratados a $500^{\circ} \mathrm{C}$. 




Figura 4. Espectros de emisión normalizados para los recubrimientos de sílice preparados a partir de (๑) TMOS, (O) TEOS y (コ) TMOS+MTES, densificados a distintas temperaturas (curvas desplazadas).

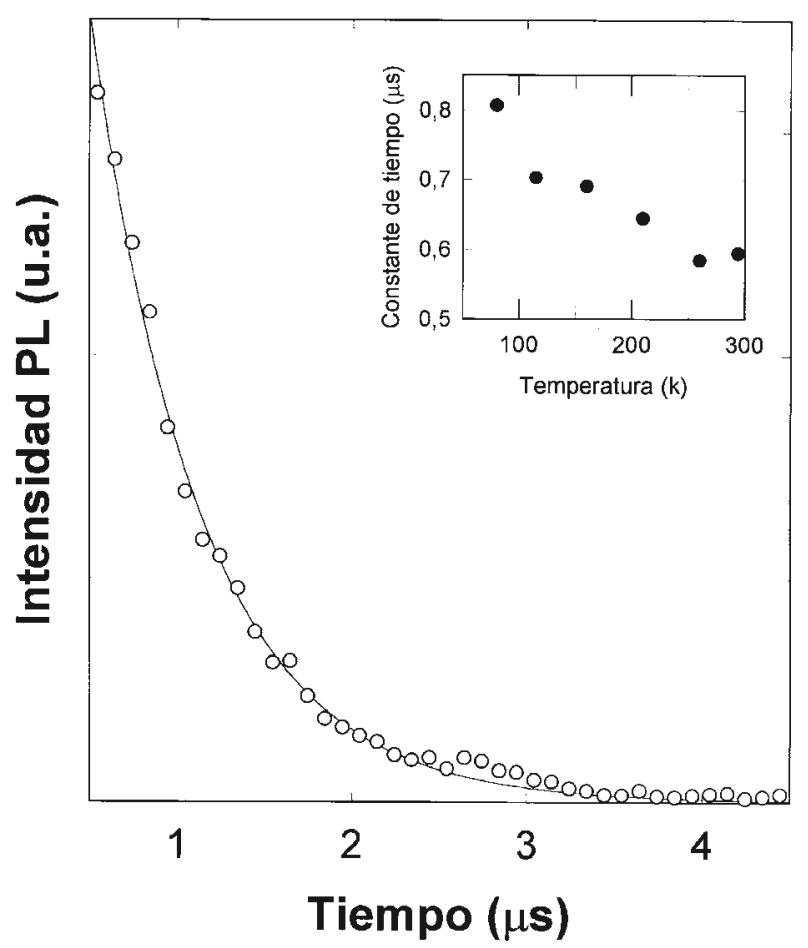

Figura 5. Caída temporal de la luminiscencia a temperatura ambiente para un recubrimiento de sílice correspondiente a una energía de excitación de 4,66 eV (266 nm) con un láser de Nd:YAG y la emisión de 3,0 $\mathrm{eV}(410 \mathrm{~nm})$. El resultado experimental $(\bigcirc)$ puede ser ajustado a una función exponencial con constante de tiempo $r=0.609 \mu \mathrm{s}$. El detalle muestra la evolución de la constante de tiempo con la temperatura $(\bullet)$.
4,66 eV (266 nm), utilizando como fuente de exitación un láser de Nd:YAG. El resultado fue una caída exponencial (ver figura 5) con constante de tiempo a temperatura ambiente de $r=0.61 \mu \mathrm{s}$. Para las medidas realizadas a distintas temperaturas, la caída mantiene la forma exponencial, con una constante de tiempo que decrece con la temperatura, como se muestra en el recuadro intercalado de la figura 5 .

\section{DISCUSIÓN}

Como ya se ha mencionado en la introducción, existe un gran número de defectos ópticamente activos en la sílice [2]. Estos defectos se clasifican en dos grandes grupos: los defectos que están relacionados con una deficiencia de oxígeno (DDO) como centros de tipo $\mathrm{E}^{\prime}, \mathrm{B}_{2}$ o enlaces $\mathrm{Si}-\mathrm{Si}$, y los relacionados con el exceso de oxígeno (DEO) como radicales peróxido, enlaces $\mathrm{O}=\mathrm{O}$ o grupos silanol. Asimismo, hay un gran volumen de información sobre centros ópticos de la sílice, debidos a la presencia de impurezas. En nuestro caso, el hecho de que la emisión luminiscente a 3,4 eV (365 nm) sea independiente del precursor utilizado y de la presencia de pequeñas cantidades de iones metálicos, indica un origen independiente de posibles impurezas residuales. El origen de la luminiscencia debe estar, por tanto, asociado con algún tipo de defecto relacionado con los formadores de red. Además, al ser la intensidad de dicha luminiscencia independiente del espesor de los recubrimientos, nos induce a pensar que probablemente se trate de un defecto que se encuentra localizado en la región superficial de la muestra; pues si se encontrara en el volumen, la intensidad de la emisión debería variar con el espesor del recubrimiento, esto es, con la concentración de defectos excitados. Por otro lado, los resultados indican que cuando los recubrimientos se exponen a una atmósfera reductora (preparación en cámara de atmósfera controlada o tratamiento térmico en atmósfera de $\mathrm{N}_{2}-\mathrm{H}_{2}$ ) y, por tanto deficiente en oxígeno, la intensidad de la luminiscencia a 3,4 eV (365 $\mathrm{nm}$ ) disminuye. Este comportamiento sugiere un origen de la luminiscencia relacionado con un exceso de oxígeno, esto es, un defecto de tipo DEO. En este sentido cabe señalar que la posición de la banda de excitación de la emisión a 3,4 eV coincide con la banda de absorción debida a enlaces de tipo peróxido ( $=$ Si-O-O-Si $\equiv$ ) observada en sílice sintética de alta pureza [14], lo que avala esta última hipótesis. Además, las características de la emisión son también coherentes con un origen debido a grupos silanol $(=\mathrm{Si}-\mathrm{OH})$ asociados a las moléculas de agua que se encuentran en los poros superficiales en este tipo de recubrimientos. Así, los débiles enlaces $\mathrm{H}$ (de hidrógeno) entre las moléculas de agua adsorbidas en la superficie y los grupos silanol podrían producir una débil red de átomos de oxígeno y silicio similar a los de tipo peróxido.

Cuando se trata el recubrimiento a una temperatura de $500^{\circ} \mathrm{C}$, la variación observada en la componente de la emisión a 3 eV, sugiere un origen relacionado con grupos metilo, ya que a esta temperatura comienzan a eliminarse del recubrimiento. Esto estaría de acuerdo con la aparición y aumento de una banda de catodoluminiscencia en $2.99 \mathrm{eV}(415 \mathrm{~nm})$ que aparece en sílice pura implantada con átomos de carbono [15]. Por otro lado, los defectos de tipo O-O o de tipo $\mathrm{O}_{2}$ también se han relacionado con emisiones en la zona comprendida entre 3,1 y 2,9 eV (390 y $430 \mathrm{~nm}$ ) [16,17], por lo que habría que tenerlos en cuenta como posibles candidatos de la componente de $3 \mathrm{eV}$. La ausencia de bandas en el espectro de absorbancia no es significativa, pues en la bibliografía existente se encuentra que la mayoría de las bandas de absorción relacionadas con defectos de sílice se encuentran a energías mayores de $4 \mathrm{eV}$ [2]. 


\section{CONCLUSIONES}

El estudio espectroscópico de los recubrimientos de sílice resulta un elemento interesante para su caracterización. El análisis de los resultados pone de manifiesto que el origen de la luminiscencia esta íntimamente ligado al proceso de densificación y la formación de la red vítrea. Por ello este tipo de estudios puede ser de gran utilidad en el conocimiento de dicho proceso, ya que supone un método no destructivo.

Los recubrimientos sol-gel estudiados presentan un pico de luminiscencia a 3,35 eV (370 nm) junto con otro pico secundario alrededor de 2,95 eV (420 nm), al excitar con luz de 3,85 eV (322 nm). El pico principal de 3,35 eV se relaciona con grupos silanol y/o moléculas de agua adsorbida a los silanoles en las zonas más superficiales de los recubrimientos. Por otro lado, la presencia de defectos tipo carbono y/o oxígeno está probablemente implicada en el proceso luminiscente de la emisión de 2,95 eV. La forma del espectro de emisión es independiente del prescursor utilizado para aportar la sílice.

\section{AGRADECIMIENTOS}

Los autores agradecen al Dr. M. Aparicio las medidas de espesor de los recubrimientos con perfilómetro, así como al Prof. Polloni, a la Dra. Borsella y a A. dal Vecchio la ayuda prestada con las medidas de caída temporal de la luminiscencia. Asimismo, M.A.G. agradece al Prof. Mazzoldi de la Universidad de Padua la hospitalidad dispensada durante su estancia en dicho centro.

Este trabajo ha sido parcialmente financiado por los proyectos PB97-0287 de la DGES y 2FD97-0141 de la CE-FEDERCICYT.

\section{BIBLIOGRAFIA}

1. "Sol-Gel Optics: Processing and Applications" editado por L.C. Klein. Kluwer Acad. Pub. Norwell, 1994. Cáp. 6.

2. L. Skuja. “Optically active oxygen -deficiency-related centers in amorphous silicon dioxide". J. Non-Crsyt. Solids 239, 16-48 (1998).

3. H. Imai y H. Hirashima. "Intrinsic-and extrinsic-defect formation in silica glasses by radiation". J. Non-Cryst. Solids 179, 202-213 (1994).
4. E. J. Friebele, D.L. Griscom y M. Stapelbroek. "Fundamental defect centers in glass: The peroxy radical in irradiated, high purity, fused silica". Phys. Rev. Lett. 42, 1346-1349 (1979).

5. U. Kreibig y M. Vollmer. "Optical properties of Metal Clusters". SpringerVerlag. Berlin (1995).

6. P. Mazzoldi, G.W. Arnold, G. Battaglin, F. Gonella y F. Hauglund Jr. "Metal nanocluster formation by ion implantation in silicate glasses: Nonlinear optical applications". J. Non-Linear Optical Phys. and Mater. 5, 285-330 (1996).

7. G. De Marchi, F. Gonella, P. Mazzoldi, G. Battaglin, E.J. Knystautas y C. Meneghini. "Non-linear glasses by metal cluster formation: synthesis and properties". J. Non-Cryst. Solids 196, 79-83 (1996).

8.S.E.Paje,J.Llopis, M.A.VillegasyJ.M.FernándezNavarro. “Photoluminescence of a silver-doped glass". Appl. Phys. A 63, 431-434 (1996).

9. M.A. Villegas, M.A. García, J. Llopis y J.M. Fernández Navarro. “Optical spectroscopy of hybrid sol-gel coatings doped with noble metals". J. Sol-Gel Sci. Techn. 11, 251-265 (1998).

10. P. Boutinaud y H. Bill. “Optical centers related to silver in $\mathrm{Ag}^{+}$-doped strontium fluoride crystals". J. Phys. Chem. Solids, 57, 55-64 (1996).

11. T.Tani, H. Namikawa, K. Arai y A. Makashima. "Photochemical hole-burning study of 1,4-dihydroxyanthraquinone doped in amourphous silica prepared by alcoholate method". J. Appl. Phys. 58, 3559-3565 (1985).

12. D. Avnir, D. Levy y R. Reisfeld. "The nature of the silica cage as reflected by spectral changes and enhanced photostability of trapped rhodamine $6 \mathrm{G}^{\prime \prime}$. J. Phys. Chem. 88, 5956-5959 (1984).

13. E. Borsella, F. Gonella, P. Mazzoldi, A. Quaranta, G. Battaglin y R. Polloni. "Spectroscopic investigation of silver in soda-lime glass". Chem. Phys. Letters 284, 429-434 (1998).

14. H. Nishiwaka, R. Tohmon, Y. Ohki, K. Nagasawa y Y. Hama. “Defect and absoption bands induced by surplus oxygen in high-purity synthetic silica". J. Appl. Phys. 65, 4672-4678 (1989).

15. H. Koyama. “Cathodoluminescence study of $\mathrm{SiO}_{2}$ ”. J. Appl. Phys. 51, 22282235 (1980).

16. H. Nishikawa, Y. Shiroyama, R. Nakamura, Y. Ohki, K. Nagasawa y Y. Hama. "Photoluminescence from defect centers in high purity silica glasses observed under 7.9 -eV excitation". Phys. Rev. B 45, 586 (1992).

17. T.D.S. Hamilton, I.H. Munro and G. Walker. "Luminescence Spectroscopy" editado por M.D. Lumb Academic, London 1978. Cáp. 3.

Recibido: 13-1-99

Aceptado: 18-9-99 